\title{
Théologiques
}

\section{Questions à la théologie chrétienne après la Shoah}

\section{Dominique Cerbelaud}

Volume 11, numéro 1-2, automne 2003

Juifs et chrétiens. L’à-venir du dialogue.

URI : https://id.erudit.org/iderudit/009536ar

DOI : https://doi.org/10.7202/009536ar

Aller au sommaire du numéro

Éditeur(s)

Faculté de théologie de l'Université de Montréal

ISSN

1188-7109 (imprimé)

1492-1413 (numérique)

Découvrir la revue

Citer cet article

Cerbelaud, D. (2003). Questions à la théologie chrétienne après la Shoah.

Théologiques, 11(1-2), 271-283. https://doi.org/10.7202/009536ar d'utilisation que vous pouvez consulter en ligne.

https://apropos.erudit.org/fr/usagers/politique-dutilisation/ 


\title{
Questions à la théologie chrétienne après la Shoah
}

\author{
Dominique Cerbelaud \\ Faculté de théologie \\ Université catholique de Lyon
}

Si le choc de la Shoah concerne au premier chef la tradition juive, où il a suscité des interrogations et des remises en cause parfois radicales, on peut estimer qu'il a aussi provoqué des ébranlements dans la théologie chrétienne. Celle-ci se trouve en effet contrainte à un sérieux examen de conscience: qu'en est-il de l'antijudaïsme qu'elle a si longtemps secrété ? Quelle relation peut-on établir entre cet antijudaïsme et l'antisémitisme moderne, voire l'antisémitisme nazi? Plus largement, quel sens ce déferlement du mal peut-il recevoir dans l'histoire humaine? Ne remet-il pas en cause cette histoire elle-même, comme lieu de révélation du Salut ${ }^{1}$ ?

Mais au-delà de ces secousses directes, le séisme de la Shoah ne cesse de provoquer contrecoups et répliques. Dans la mesure où la théologie chrétienne s'est pour une part construite sur une délégitimation de l'existence d'Israël, c'est par pans entiers qu'elle se trouve, de proche en proche, «revisitée ${ }^{2} »$ d'un œil critique. En effet, c'est la «théologie de la substitution » elle-même qui se trouve aujourd'hui récusée, sous toutes ses formes et dans tous ses prolongements. L'évaluation de ce séisme s'avère difficile: comment mesurer l'impact d'une telle mutation, souvent indirect, voire implicite? Il est cependant possible de risquer quelques

1. Dans ce sens, voir déjà B. Dupuy, «La théologie chrétienne après la Shoah» dans Istina, 36 (1991) p. 291-307.

2. L'expression est d'A. Blancy, dans un article qui esquisse un premier repérage de ces remises en question: "La théologie chrétienne d'après la Shoah» dans Foi \& Vie, 99 (février 2000) p. 63-80. Voir notamment p. 72; voir aussi, du même auteur: "Théologie chrétienne post-Shoah» dans Sens, nouv. série, 53, n 261 (2001) p. 405-416. 
"coups de sonde». C'est le but du présent article, qui s'arrêtera sur un certain nombre de questions, en s'appuyant pour chacune d'elles sur le travail d'un auteur particulier. Je ne me dissimule, ce faisant, ni le risque de procéder à des choix subjectifs, voire arbitraires, ni celui de tracer l'image d'un paysage quelque peu «impressionniste». Mais il me semble que nous sommes encore loin de pouvoir proposer, dans ce domaine, une synthèse exhaustive: la déflagration de la Shoah n'a pas encore, loin s'en faut, produit tous ses effets. On peut même estimer qu'elle ne fait que commencer à les révéler...

\section{Nouvelle approche herméneutique du Nouveau Testament}

L'une des principales "corrections» induites par le congédiement de la théologie de la substitution a consisté à redécouvrir la judaïé de Jésus et des figures fondatrices du christianisme, et plus largement à aborder le Nouveau Testament non plus «depuis l'aval» — la tradition chrétienne — mais «depuis l'amont» - la tradition juive. Certes, divers pionniers avaient ouvert la voie dans ce domaine ${ }^{3}$, mais la présentation la plus habituelle se soldait, jusqu'à une époque récente, par une "déjudaïsation » des Évangiles et des personnages qui y évoluent. C’est ainsi que les apôtres, autour de Jésus et de Marie, figuraient "la première Église "; à vrai dire un seul d'entre eux persistait à incarner le judaïsme: $\operatorname{Judas}^{4} \ldots$ De manière symptomatique, cette christianisation naïve fait aujourd'hui sourire. Plus largement, il semble que la volonté de resituer le texte fondateur du christianisme sur son horizon réel, celui du judaïsme du $\mathrm{I}^{\mathrm{er}}$ siècle de notre ère, fasse désormais partie intégrante de l'exégèse néotestamentaire comme telle.

Simultanément, la connaissance du monde juif de cette époque a enregistré depuis quelques décennies des progrès considérables, en particulier du fait de la découverte de Qumrân, et d'une approche plus systématique des sources rabbiniques anciennes. Dès lors, c'est sur une

3. Sans remonter à R. Simon et à sa préface à l'ouvrage du rabbin L. de Modène, Cérémonies et coutumes qui s'observent aujourd'bui parmi les Juifs (Paris, 1674), citons J. Bonsinven, Textes rabbiniques des deux premiers siècles chrétiens, Rome, Pontificio Istituto Biblico, 1955; H. Strack et P. Billerbeck, Kommentar zum Neuen Testament aus Talmud und Midrasch, Munich, Beck, $1978^{6}$ (1922-1960).

4. Sur ce dernier point, voir par exemple Augustin, Enar. in Psalm 108,1. 
connaissance renouvelée du «monde où vivait Jésus ${ }^{5}$ » que l'on peut désormais s'appuyer.

Parmi les auteurs qui arpentent ce domaine, je citerai volontiers le réformé hollandais Peter Tomson, dont le maître livre vient tout juste d'être traduit en français ${ }^{6}$. Nous y trouvons une excellente synthèse de cette nouvelle approche herméneutique des plus anciens documents chrétiens, que je me propose de présenter ici brièvement.

L'auteur, qui se situe explicitement "après Auschwitz ${ }^{7}$ ", propose de saisir le Nouveau Testament comme une Écriture juive. Mais il convient tout d'abord de dresser la carte des divers courants, sensibilités et tendances du judaïsme du I $^{\text {er }}$ siècle de notre ère - en tirant profit du renouvellement des connaissances à ce sujet, que l'on vient de mentionner.

Comment Jésus lui-même s'est-il situé dans ce contexte? La question en fait immédiatement surgir une autre, actuellement fort débattue: celle de l'accès possible au «Jésus historique ». L'auteur y propose sa propre réponse et s'efforce de préciser ce qu'il en est de l'enseignement et de la pratique de Jésus, de son rapport à la Torah et aux différents groupes juifs (sans oublier les non juifs).

Peter Tomson examine ensuite le corpus lucano-paulinien (qu'il juge dans l'ensemble bienveillant à l'égard du judaïsme, notamment pharisien); les Évangiles de Marc et de Matthieu (dont la rédaction complexe, en particulier pour ce dernier, aboutit à faire voisiner les accents les plus vivement judéo-chrétiens avec de vigoureuses polémiques anti-pharisiennes); le quatrième Évangile et les épîtres johanniques (émanant d'un milieu proche des sadducéens, mais dont les dernières couches rédactionnelles témoignent d'un véritable antijudaïsme); enfin les «lettres des Églises juives» (incluant la Didachè), à la tonalité beaucoup plus nettement judéo-chrétienne, voire anti-paulinienne. Chemin faisant, son ouvrage aborde des problèmes intimement liés à celui de la rédaction — en particulier celui de la formation du «Canon» des Écritures chrétiennes. Ce status quaestionis suscitera sans aucun doute des discussions sur tel ou

5. Pour reprendre le titre du bel ouvrage collectif paru sous la direction de H. Cousin, Paris, Cerf, 1998.

6. P. Tomson, Jésus et les auteurs du Nouveau Testament dans leur relation au judaïsme, Paris, Cerf, 2003.

7. Voir notamment p. 21. 
tel point de détail. Mais sa publication constitue à elle seule, me semblet-il, un événement dans l'histoire de l'exégèse du Nouveau Testament.

\section{Du Jésus juif au Christ des confessions de foi}

Cette nouvelle approche du Nouveau Testament suscite une question inédite: si l'on accepte que s'y dessine un portrait de Jésus «intégralement juif », comment la christologie traditionnelle pourra-t-elle accepter un tel point de départ? Autrement dit, comment s'articulent aujourd'hui "Jésus » et "Christ" ? Déjà énoncée de façon polémique par les auteurs des "vies de Jésus" du XIX ${ }^{\mathrm{e}}$ siècle, la question n'a fait depuis lors que s'aggraver, notamment dans le contexte de l'actuelle «troisième quête » du Jésus historique.

C'est une catholique états-unienne, Rosemary Radford Ruether, qui a formulé la question en termes radicaux, voire provocateurs, dans le livre qu'elle a consacré à la critique théologique de l'antijudaïsme chrétien : l'antijudaïsme ne représente-t-il pas «l'autre face» de la christologie dogmatique ${ }^{8}$ ? Connue comme théologienne féministe, cette auteure aperçoit des liens entre la reconnaissance des femmes et celle du judaïsme dans une théologie chrétienne massivement masculine et anti-juive ${ }^{9} \ldots$ Mais ce serait là un autre aspect de notre sujet.

En ce qui concerne le plan dogmatique, R.R. Ruether estime que l'antijudaïsme de l'affirmation christologique provient essentiellement d'une accentuation de l'affirmation de l'accomplissement: l'eschatologie s'inscrit dans l'histoire, de sorte qu'il n'y a plus lieu d'y attendre quelque avènement que ce soit. Cette "eschatologie réalisée» transforme les binômes bibliques (jugement et promesse; particularisme et universalisme; lettre et esprit; histoire et eschatologie) en expressions dualistes, dont les chrétiens s'arrogent systématiquement le «pôle positif» et appliquent

8. R.R. Ruether, Faith and Fratricide: The Theological Roots of Anti-Semitism, New York, Seabury Press, 1974. Voir notamment les p. 246-251 (\$ intitulé: “The Key Issue: Christology”). C'est là qu'elle utilise la formule que je citais à l'instant: «Our theological critique of Christian Anti-Judaism, therefore, must turn to what was always the other side of anti-Judaism, namely Christology.» (p. 246)

9. Voir notamment son article: «Feminism and Jewish-Christian Dialogue. Particularism and Universalism in the Search for Religious Truth" dans J. Hick et P. Knitter, dir., The Myth of Christian Uniqueness, Londres, $1988^{2}$ (1 $1^{\mathrm{re}}$ éd.: Maryknoll, New York, 1987), p. 137-148. 
l'autre à Israël. Fort heureusement, cette affirmation du «déjà-là » laisse une certaine place, dans la spiritualité chrétienne, au "pas-encore»: l'inaccomplissement se loge dans l'accomplissement lui-même. De ce point de vue, le christianisme a tout intérêt à comprendre et à développer sa proximité avec le judaïsme, qui pour sa part affirme la priorité du "pas-encore » sur le «déjà-là ».

On pourrait, à vrai dire, aller beaucoup plus loin dans cette direction - que notre auteure ne fait ici qu'ébaucher. À la réflexion en effet, c'est tout l'ensemble du processus de dogmatisation de la figure du Christ qui pourrait venir en question: n'a-t-il pas nécessairement pour effet de "déshistoriciser ", et dans le cas présent de déjudaïser, son point de départ? On l'a souvent fait remarquer à propos de la formule de foi de Chalcédoine, mais cela vaut aussi pour les Symboles de foi de l'époque patristique, et peut-être même déjà pour les passages du Nouveau Testament où s'ébauche la «christologie sapientielle»! Ultimement, c'est à une réflexion sur le clivage entre la notion sémitique de "messie» et celle, grecque, de «christ», que l'on se trouvera conduit tôt ou tard. Indiquons-le au passage: cette relecture critique n'implique en aucune façon un abandon. On voit mal comment la tradition chrétienne pourrait renier sa propre élaboration! En revanche, on voit bien comment elle peut y apporter des infléchissements, des correctifs et des relativisations.

\section{Une théologie de la Croix après Auschwitz?}

S'il est un domaine de la dogmatique chrétienne qui se trouve très directement et très violemment ébranlé par la déflagration d'Auschwitz, c'est bien celui de la sotériologie, ou doctrine du Salut. Pour la théologie classique en effet, notamment depuis saint Anselme de Cantorbéry au XI siècle, il y a une sorte d'équivalence entre «souffrance» et «Salut ». Dans le cas de l'homme-Dieu, cette souffrance juxtapose et harmonise devoir et pouvoir: l'homme «devait» satisfaire à l'immense colère de Dieu mais ne le pouvait pas; Dieu «pouvait» le faire mais ne le devait pas. La souffrance d'un homme-Dieu résout cette aporie ${ }^{10}$.

Dès lors, la tentation sera grande d'identifier purement et simplement souffrance et Salut. Et cela aura pour conséquences, d'une part d'identifier la mort de Jésus en croix comme «moment salutaire» principal —

10. Voir notamment A. de Cantorbéry, Cur Deus homo, I, 19-20; II, 6-7. 
pour ne pas dire unique; d'autre part, de valoriser la souffrance chrétienne comme un moyen de «s'unir» à la Passion salutaire. Dans les formes populaires de jansénisme catholique, cela conduira à admettre qu' «il faut faire des sacrifices». Corollairement, si toute souffrance apparaît comme "une croix", tout plaisir s'assimile à un péché. On reconnaît ici la nébuleuse thématique qui a commandé la spiritualité catholique courante jusqu'au début du $\mathrm{xx}^{\mathrm{e}}$ siècle...

C’est précisément le choc de la Shoah qui a contribué, explicitement ou implicitement, à la désagréger. Comment osera-t-on reconnaître une valeur positive à la mort de six millions de justes et d'innocents appartenant au Peuple élu? Seul un Dieu pervers - pour ne pas dire sadique - pourrait y trouver une quelconque gratification! Et si l'on reconnaît l'absurdité d'un tel événement, alors comment continuer à reconnaître l'histoire humaine comme une "histoire du Salut»?

Selon son habitude, la théologie chrétienne aura tendance à subsumer cette souffrance dans la croix de Jésus, par exemple en qualifiant Auschwitz de "Golgotha du monde contemporain ${ }^{11}$ ». Mais une telle herméneutique révèle vite ses limites.

Pour décrire ces dernières, je m'appuierai volontiers sur un article du carme Michel de Gœdt, paru pro manuscripto dans les publications du Centre chrétien pour l'étude du judaïsme (Lyon): «Penser la théologie de la rédemption auprès Auschwitz ${ }^{12} »$. Dans ce texte remarquable, l'auteur propose en effet un renversement de perspective assez radical.

Deux «figures» bibliques permettent selon lui de typologiser l'antijudaïsme: celle du pharaon et celle d'Amaleq. Si le premier représente la forme habituelle (si l'on ose dire!) de l'antisémitisme, le second renvoie à une hostilité autrement plus fondamentale: une volonté d'ex-

11. L'expression a été employée par Jean-Paul II lors de son voyage en Pologne de juin 1979: voir Documentation Catholique, $\mathrm{n}^{\mathrm{o}} 1767$ (1 ${ }^{\text {er }}$ juillet 1979) p. 632. Elle a provoqué des réactions parfois vives, notamment dans le monde juif.

12. Voir Choix de textes de recherche théologique judéo-chrétienne, $\mathrm{n}^{\circ} 2$ (sans date) p. 22-50. Ce cahier comporte deux articles de M. de GÆDt. Mais si le premier, «La véritable "question juive" pour les chrétiens : celle qui les met eux-mêmes en question ", a fait l'objet d'une reprise (voir M. de GÆDT, "La véritable "question juive” pour les chrétiens - Une critique de la théologie de la substitution » dans Nouvelle Revue Théologique, 114 [1992], p. 237-250), il n'en va pas de même, à ma connaissance, du second, qui reste donc aujourd'hui fort difficile à trouver... 
termination d'Israël «en chemin vers la Terre promise », volonté que l'on peut reconnaître dans l'antisémitisme nazi.

Récusant toute comparaison entre la souffrance de la Shoah et celle de Jésus au Golgotha, Michel de Gœdt fait remarquer que cette dernière ne constitue en aucune façon un extrême, ni par sa teneur ni en raison de quelque sensibilité particulière de Jésus. Ce qui reste à scruter, c'est le sens que ce dernier a conféré à sa Passion, ou plus exactement l'opacité qu'il y a endurée. Comme le rappelle l'épître aux Hébreux, c'est une compassion particulière qui se découvre ici (cf. He 5,7-10). Qu'est-ce qui interdit d'étendre cette compassion de Jésus à la souffrance juive de notre temps?

L'humilité de la Rédemption, c'est peut-être ceci : accepter d'être, non point un passage triomphal, mais un point parmi d'autres: fluette histoire qui pâlit d'être noyée dans le chaos des monstruosités de l'Histoire, mais fluette histoire d'un homme attristé jusqu'à la mort de voir tant de souffrances chez ceux dont il se fait le frère et compagnon, d'un homme humblement solidaire de cette souffrance ${ }^{13}$.

Il convient, me semble-t-il, d'accueillir avec beaucoup d'attention cette tentative pour repenser la croix de Jésus: elle pourrait éveiller des échos inattendus chez d'autres auteurs, tels que Hans Jonas (qui, dans Le concept de Dieu après Auschwitz, plaide pour une nouvelle compréhension de l'être divin et de son rapport à l'histoire) ou Etty Hillesum (qui, dans son magnifique journal paru sous le titre français Une vie bouleversée, développe l'intuition selon laquelle c'est à nous désormais d'aider Dieu, et non plus l'inverse)...

\section{Retrouvailles avec Israël et œcuménisme chrétien}

Dans une autre direction, il convient de s'interroger sur le lien entre les retrouvailles avec Israël et le dialogue œcuménique interne au christianisme. Non pas certes qu'en revenant à leur origine juive les Églises retrouveraient un «degré zéro" du christianisme susceptible de constituer leur base commune: on ne saurait en aucune façon effacer la réalité de l'histoire des diverses entités chrétiennes séparées! Mais il s'agit de scruter l'analogie entre le «schisme» qui sépare l'Église naissante de la Synagogue et ceux qui se sont produits au sein du christianisme. On a pu faire

13. De GÆDt, «La véritable “question juive” ", p. 44. 
remarquer que, dans toutes les confessions chrétiennes, la présentation de l'histoire (en catéchèse, en prédication, dans les manuels de théologie...) prenait facilement l'allure d'une autojustification, les "autres» faisant figure de déviants auxquels, depuis leur dissidence, il n'y a plus lieu de s'intéresser: c'est le même traitement que l'on réserve à Israël depuis Jésus! Plus profondément encore, on peut s'interroger sur le mécanisme de toutes ces séparations: les schismes intrachrétiens ne répètent-ils pas le «schisme » originel, celui qui a éloigné l'Église de la Synagogue — une rupture d'autant plus encline à se réitérer qu'elle a toujours été occultée par la théologie chrétienne?

C’est encore un théologien réformé, français cette fois, qui a posé avec le plus de vigueur et de précision cette question. Il s'agit de Fadiey Lovsky, notamment dans l'un des ouvrages qu'il a consacrés aux relations judéo-chrétiennes ${ }^{14}$. Plus précisément, c'est dans le chapitre 7 de ce livre, intitulé "Le peuple d'Israël, pivot œuménique du peuple de Dieu », que l'auteur développe cette problématique. Il commence par rappeler que c'est l'amour de Dieu qui «fait» l'élection, celle d'Israël comme celle des nations dans l'Église. L'antijudaïsme, tout comme les guerres de religion intrachrétiennes, ne peuvent relever que d'une violence humaine. Or, de manière symptomatique, les Églises chrétiennes n'ont cessé, au cours de l'histoire, de théologiser leur «rejet» les unes des autres, tout comme elles avaient théologisé leur «rejet» d'Israël. C'est donc à Dieu lui-même que l'on attribuera ces abandons successifs de toutes les communautés croyantes... à l'exception, bien entendu, de celle à laquelle appartient celui qui parle!

Dès lors, il se pourrait bien que la conversion à une «théologie de l'estime " à l'égard d'Israël ait pour effet une véritable guérison des relations entre chrétiens: l'autre a autant de droit à exister que moi-même, puisque son existence et la mienne viennent d'une seule et même volonté de Dieu.

Notons que l'auteur ici mentionné maintient la perspective «classique» selon laquelle l'unité des chrétiens est solidaire de la conversion d'Israël, c'est-à-dire de la reconnaissance, par ce peuple de la première Alliance, de l'accomplissement des promesses en Jésus-Christ. Reprenant

14. F. Lovsкy, La déchirure de l'absence. Essai sur les rapports entre l'Église du Christ et le peuple d'Israël, Paris, Calmann-Lévy, 1971. 
le mot $\pi \rho o ́ \sigma \eta \mu \psi \imath \varsigma$ de Rm 11,15, Michel de Gœedt s'interdisait pour sa part d'en dire plus sur cette «réintégration ». Du reste, d'autres exégètes font remarquer aujourd'hui que $\mathrm{Rm}$ 11,26 évoque le salut d'Israël et non sa conversion...

\section{L'unique Alliance et son «relief interne»}

Restons-en à l'exégèse, cette fois pour tenter d'élucider une question-clé du dialogue judéo-chrétien: celle de l'Alliance. Dans le langage chrétien courant, en effet, il est question de l' «Alliance nouvelle», qui en vient toujours peu ou prou à supplanter l'" ancienne Alliance ", celle que Dieu avait nouée avec Israël. Une telle compréhension apparaît donc directement solidaire de la théologie de la substitution.

Or, il se trouve que le pape Jean-Paul II, dans un discours prononcé à Mayence le 17 novembre 1980, faisait allusion à cette première Alliance, «une Alliance jamais révoquée par Dieu». Prononcée en allemand, la formule devait dans un premier temps faire l'objet de méprises et d'incompréhensions fort symptomatiques: la traduction italienne comporte à cet endroit un contresens ${ }^{15}$, tandis que la traduction française, parfaitement inintelligible, suscitera un correctif ultérieur ${ }^{16}$.

Sur ces entrefaites, l'exégète allemand Norbert Lohfink s'est livré à une étude approfondie de ce thème scripturaire. Son petit livre, qui prend pour titre la «Formule de Mayence» elle-même ${ }^{17}$, constitue un remarquable status quaestionis. En douze petits chapitres, il montre tout d'abord l'enjeu de la question: doit-on estimer qu'il y a une seule Alliance, deux Alliances ou une pluralité d'Alliances ${ }^{18}$ ? Il scrute ensuite divers textes bibliques, essentiellement 2 Co 3,14 (avec sa mention de

15. Voir J. STERN, "Jean-Paul II face à l'anti-judaïsme" dans Radici dell'antigiudaismo in ambiente cristiano, Colloquio intra-Ecclesiale, Atti del Simposio teologico-storico, Città del Vaticano, 1 novembre 1997 (Atti e documenti 8), Rome, Libraria Editrice Vaticana, 2000, p. 54-78, spécialement p. 66 et note 37.

16. Texte dans Documentation Catholique, $\mathrm{n}^{\circ} 1798$ (21 décembre 1980) p. 1148; correctif dans Documentation Catholique, $\mathrm{n}^{\circ} 1807$ (3 mai 1981) p. 427.

17. N. Lohfink, Der niemals gekündigte Bund, Freiburg im Breisgau, Herder, 1989.

18. "Vielleicht kann man dann auch leichter und mit Angabe von Gründen zwischen den auf dem theologischen Dialog-Markt angebotenen verschiedensten, Ein-BundTheorien', Zwei-Bünde-Theorien' und,Viele-Bünde-Theorien' unterscheiden », LoHFInk, Der niemals gekündigte Bund, p. 24. 
l'«ancienne Alliance»), l'épître aux Hébreux, Jr 1 et Rm 9-11. Il fait notamment remarquer que le premier de ces passages ne fait aucune référence au livre de Jérémie, et qu'il n'y a donc pas lieu d'y lire en filigrane le chapitre 8 de l'épître aux Hébreux - comme on l'a presque toujours fait. Le texte d'He 8, fortement marqué par une théologie alexandrine platonisante, vise d'ailleurs en réalité le culte du Temple. À l'issue de cet examen, Lohfink conclut qu'il n'y a bien qu'une seule et unique Alliance, mais qui a donné lieu à deux voies de Salut (ch. 10). On peut se demander si cette contribution décisive a reçu toute l'attention qu'elle méritait.

\section{Jésus-Christ seul médiateur?}

S'il en est bien ainsi, alors une autre question ne tarde pas à se profiler: qu'en est-il de l'affirmation néotestamentaire de Jésus-Christ comme unique médiateur du Salut? À n'en pas douter, divers versets du Nouveau Testament, que l'on peut qualifier d' "exclusivistes", laissent entendre cette unicité (citons par exemple Jn 3,5; 14,6; Ac 4,12; 1 Tm 2,5). Quoi qu'il en soit du sens que l'exégèse d'aujourd'hui peut leur conférer, ces versets ont bel et bien alimenté une sotériologie "exclusiviste ", notamment dans la tradition catholique: qu'il suffise de rappeler à cet égard l'interprétation la plus habituelle de l'adage: «Hors de l'Église, point de Salut! » Mais si l'Alliance avec Moïse n'a jamais été révoquée par Dieu, il faut dès lors admettre qu'elle constitue une voie de Salut en dehors de cet "unique médiateur » qu'est Jésus-Christ! C'est donc très directement de la réévaluation du sens de la permanence d'Israël que procède cette nouvelle question.

Pour surmonter cette aporie, je me permettrai de faire référence à une hypothèse théologique que j'ai déjà eu l'occasion de formuler ${ }^{19}$, mais que j'aimerais reprendre ici. Elle part de la constatation que la figure biblique de la Sagesse a donné lieu à deux «appropriations» jumelles: en direction de la Torah dans la tradition juive, et en direction du Christ dans la tradition chrétienne. Or ces deux figures représentent bel et bien l'une et l'autre le support de la médiation, de telle sorte que (au moins avant la

19. Voir D. Cerbelaud, Écouter Israël. Une théologie chrétienne en dialogue, Paris, Cerf, 1995, notamment ch. 2 («La Torah et le Christ»), p. 37-51, et ch. 12 («Pour une sophiologie des religions non chrétiennes»), p. 173-181. 
crise arienne), c'est la gémellité du Christ et de la Torah qui symbolise la «différence» entre christianisme et judaïsme.

Ce que je propose pour ma part, c'est de renverser la perspective, en considérant les choses, ici encore, "depuis l'amont». Si véritablement l'unique Sagesse de Dieu se diffracte sur ces deux figures jumelles, alors pourquoi ne pas reconnaître qu'elle «s'est manifestée dans la Torah et dans le $\mathrm{Christ}^{20}$ » ? Comme le laisse entendre le Psaume 61(62),12: «Une fois, Dieu a parlé, deux fois, j'ai entendu »...

En somme, c'est seulement de notre point de vue qu'il y a ici deux voies de Salut...

\section{Vers une théologie des religions non chrétiennes}

Il se pourrait d'ailleurs que ce renversement de perspective dans un sens "inclusiviste» révèle une véritable fécondité dans le domaine, aujourd'hui si assidûment exploré, de la théologie des religions non chrétiennes. En effet, ce qui vaut à titre archétypal dans la relation judéo-chrétienne peut fort bien s'appliquer à d'autres univers religieux. Si l'épître aux

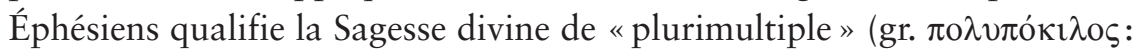
cf. Ep 3,10), il n'y a pas de raison de restreindre son champ d'activité!

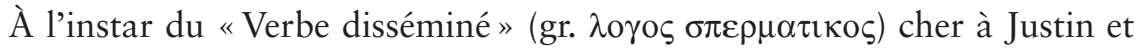
à Clément d'Alexandrie, elle se manifeste partout où les "hommes de bonne volonté» cherchent un sens transcendant à leur existence. On pourrait dès lors formuler quelque chose comme ceci : l'unique Sagesse de Dieu prend autant de formes diverses que l'histoire humaine a pu connaître de traditions religieuses...

Quoi qu'il en soit de cette piste de recherche, je voudrais citer ici quelques lignes d'un article du théologien français Claude Geffré qui énoncent excellemment, à mon sens, le caractère «archétypal » de la relation judéo-chrétienne dans le champ du dialogue interreligieux:

C'est à partir de l'irréductible d'Israël qu'on peut chercher à penser l' "irréductible" des grandes religions du monde. On peut en effet déceler une analogie entre le rapport du christianisme naissant et du judaisme et le rapport que le christianisme contemporain entretient avec les religions du monde. Il faut tenir à la fois et sans contradiction que les promesses du

20. Cerbelaud, Écouter Israël, p. 177. 
peuple de Dieu trouvent leur accomplissement dans le peuple de la Nouvelle Alliance et qu'à parler strictement l'Église ne se "substitue " pas à Israël. Il y a comme une "dilatation» du Peuple de Dieu. Cela nous aide à penser ce que peut être un accomplissement non-totalitaire. Jésus n'a pas voulu substituer une nouvelle religion à l'ancienne. Il a élargi à l'ensemble des nations païennes l'héritage qui était le monopole exclusif du peuple élu. Est-il trop téméraire de considérer le rapport de l'Église primitive et du judaïsme comme exemplaire du rapport actuel de l'Évangile aux autres religions et cultures ${ }^{21}$ ?

Sans hésiter, je réponds pour ma part à cette question : non, cela n'est pas «trop téméraire », c'est même la piste la plus prometteuse qui soit...

À travers ces sept exemples, je me suis efforcé de faire entendre l'importance et la profondeur de l'interpellation que représente, pour la théologie chrétienne, la réévaluation du sens de l'existence d'Israël: "un aide comme son encontre» (cf. Gn 2,18). En effet, les sept questions que j'ai retenues ne constituent pas des éléments mineurs, mais bien au contraire des points fondamentaux de cette tradition. Tout se passe donc comme si cette réévaluation, elle-même consécutive à la déflagration de la Shoah, l'ébranlait jusque dans ses profondeurs. Voilà qui illustre et corrobore le titre de l'une des contributions ici citées, du carme Michel de Gœedt: «La véritable "question juive" pour les chrétiens: celle qui les met eux-mêmes en question ». Insistons-y pour terminer: cette mise en question n'équivaut nullement à un anéantissement. Si déconstruction critique il y a, c'est en vue d'une nouvelle construction. En somme, il s'agit pour la conscience chrétienne de renoncer à toute perspective polémique. Si la dimension de l'" aide» prévaut sur celle de l'" encontre », alors peut advenir l'espérance d'une union féconde entre les deux protagonistes (cf. Gn 4,1)!

21. C. Gefrré, «Le fondement théologique du dialogue interreligieux " Chemins de Dialogue, 2 (juin 1993) p. 73-103 (citation de la p. 96). 


\section{RÉSUMÉ}

Consécutive à la déflagration de la Shoah, la réévaluation du sens de l'existence d'Israël conduit des théologiens chrétiens à penser autrement quelques-unes des questions les plus fondamentales de leur propre tradition: le sens du Nouveau Testament (P. Tomson), la christologie (R.R. Ruether), la sotériologie (M. de Goedt), l'oecuménisme (F. Lovdsky), l'alliance (N. Lohfink), l'unicité de la médiation de salut en Jésus-Christ (D. Cerbelaud), la théologie des religions non-chrétiennes (C. Geffré).

\section{ABSTRACT}

Because of the Shoah, the need to re-evaluate the meaning of Israel's existence pushes theologians to rethink some of the most fundamental questions of their own tradition: the meaning of the New Testament (P. Tomson), Christology (R.R. Ruether), soteriology (M de Goedt), ecumenism (F. Lovdsky), the covenant (N. Lohfink), Jesus Christ as the only mediator (D. Cerbelaud), the theology of non-Christian religions (C. Geffré). 